\title{
Contribution to the establishment of the R-banded karyotype in dogs
}

\author{
M Moreno-Millán, A Rodero, FJ Alonso, C Falcón \\ Facultad de Veterinaria, Universidad de Córdoba, \\ Laboratorio de Citogenética, Departamento de Genética, 14005 - Córdoba, Spain
}

(Proceedings of the 9th European Colloquium on Cytogenetics of Domestic Animals; Toulouse-Auzeville, 10-13 July 1990)

karyotype / R-banding / dog

The diploid number of the domestic dog (Canis familiaris $\mathrm{L}$ ) is composed of 78 chromosomes, all of them morphologically acrocentric with the exception of the $\mathrm{X}$ chromosome which is submetacentric and the metacentric $\mathrm{Y}$ chromosome.

Howard-Peebles and Pryor (1980) published the first results of R-banding in this species. Mayr et al (1983) later proposed a system of arranging R-bands. We describe a new contribution to the canine karyotype obtained after treatment with bromodeoxyuridine (BrdU).

The chromosomes of 4 dogs, 3 males and 1 female, were obtained from cultures of integral blood according to the method of de Grouchy et al (1964) slightly modified for domestic animals. R-bands were produced using a modified version of the method described by Di Berardino et al (1985): $20 \mu \mathrm{g} / \mathrm{ml}$ of BrdU under a UV lamp after staining with $1 \%$ orange acridine in phosphate buffer, $\mathrm{pH} 6.8$. Photographs were obtained with a Universal Zeiss R-III fluorescence photomicroscope on Kodalith film. Five metaphases per animal were analyzed.

Figure 1 shows a male metaphase. Figure 2 presents the idiogram with the images obtained in the R-band of the species studied.

The description of the banding of each chromosome, in accordance with the nomenclature proposed at the Paris conference, is as follows: 1: 6 positive bands separated 3-to-3 with a prominent negative band in the middle; 2: 5 bands, the most intense one is located in a central position; 3: 6 bands regularly spaced; 4: 5 positive bands, two in the first $1 / 3$ and 3 in the remaining $2 / 3$ regularly spaced, all of similar intensity; 5: 2 very intense positive bands each one located centrally in the proximal and distal halves; 6: 5 bands, the most prominent are located in the proximal $2 / 3 ; 7: 4$ positive bands regularly spaced with the 2 distal bands being more intense; 8: 4 positive bands, but the distal half is more strongly fluorescent; 9: 4 bands and one prominent negative band at the end of the proximal $1 / 3$; 10: 4 positive bands regularly spaced; 11: 1 negative band in the proximal $1 / 3$ and 3 positive bands in the distal $2 / 3 ; 12$ : 1 pale band and the end and 3 positive 


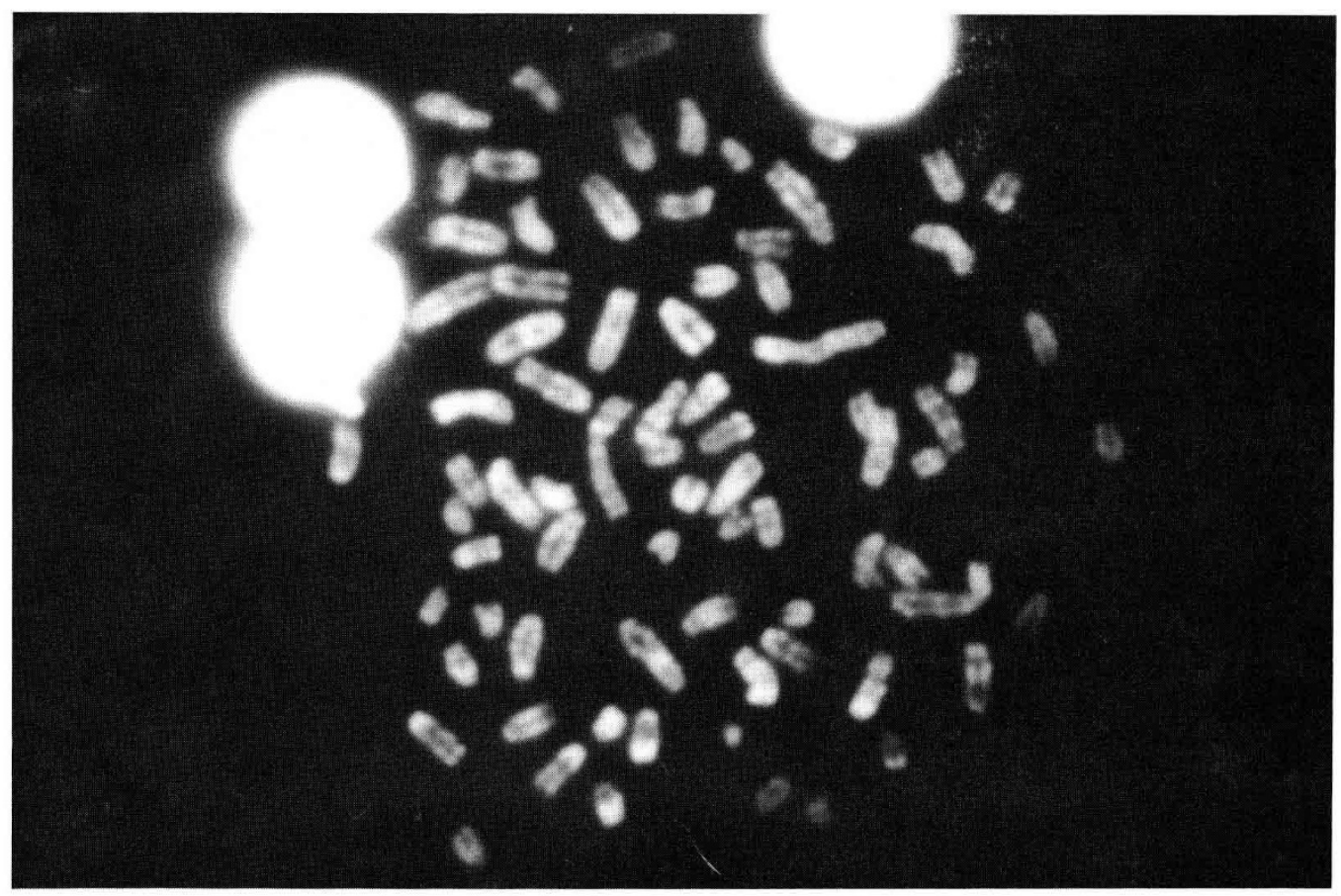

Fig 1. Metaphase plate of a male dog.

bands of similar intensity and regularly spaced; 13: proximal half very intensely stained with 2 positive bands while the distal half has 2 bands; $14: 3$ positive bands and 1 negative band at the end; 15: the distal $2 / 3$ is more intensely stained than the proximal 1/3 with 1 negative band between them; 16: 4 positive bands with the most intensely labeled one located proximally, close to the centromere; 17: 3 regularly spaced bands; 18: 3 bands, the distal band is pale; 19: 3 bands of similar intensity and regularly spaced; 20: 2 very strong positive bands in the distal half and 1 positive band in the proximal half; $21: 2$ very intense bands in the distal 2/3; 22: 3 positive bands, 1 in the proximal $1 / 3 ; 23: 3$ regularly spaced bands; $24: 2$ prominent positive bands, each one in a half; $25: 3$ positive regularly spaced bands; 26: 2 positive bands of similar intensity; 27: 3 bands with the 2 distal bands being more intense and the centromere is pale; 28: 2 positive bands with the most intense one located centrally and the centromere is pale; 29: 2 bands, the proximal band is strongly fluorescent and the centromere is pale; 30: 2 bands, 1 medium and 1 distal; 31: 2 positive bands, the proximal one being more intense than the distal one; 32: 2 very intense bands in the distal half; 33: 3 regularly spaced bands; $34: 2$ bands separated by a central, prominent negative band; 35: 1 positive band at the end; 36: 1 prominent positive band at the end; 37: 2 bands in the distal 2/3; 38: 2 bands in the distal 2/3, the most intensely labeled one is located at the end; $\mathrm{Xp}$ : 3 positive bands, the distal band is pale and the proximal band is strongly 


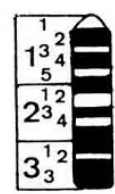

1

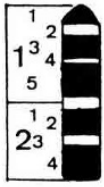

6

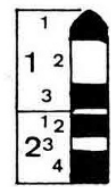

11

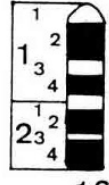

16

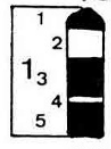

21

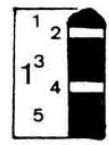

26

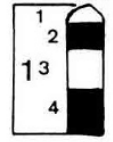

31

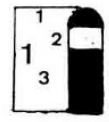

36

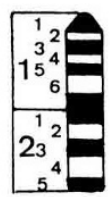

2

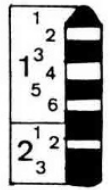

7

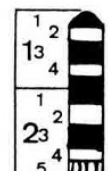

12

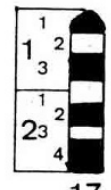

17

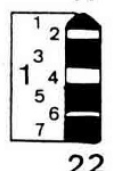

22

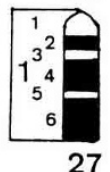

27

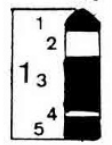

32

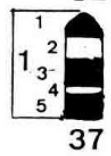

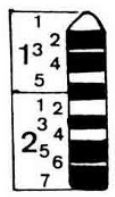

3

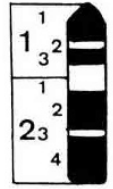

8

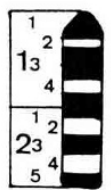

13
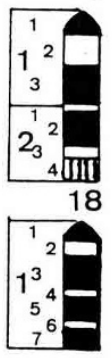

23

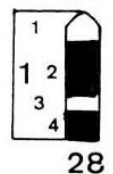

28

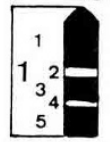

33

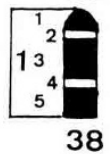

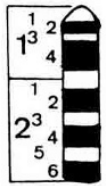

4

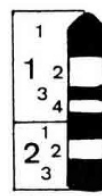

9

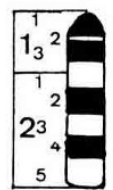

14
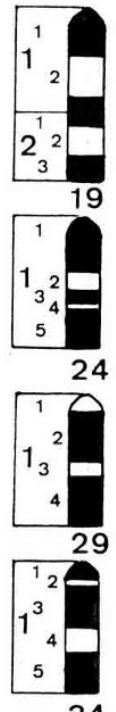

34
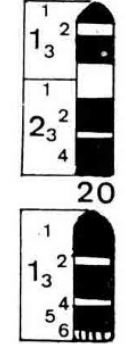

25
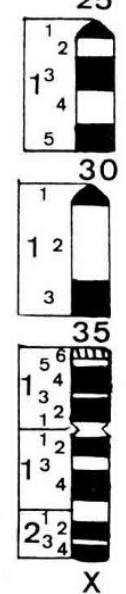

Fig 2. Idiogram illustrating the R-banding patterns of the dog. 
fluorescent; Xq: 4 bands, the central band is the most intense; Yp: 1 pale band at the end; Yq: 1 positive band at the end.

In the same way that certain standards have been studied and established for the karyotypes of other species of domestic animals, we think that it is necessary to investigate further the chromosomes of canine species to attain different objectives. On one hand, the study and characterization of different karyotypes from an evolutionary viewpoint by using high-resolution banding techniques should be envisaged; on the other, the effect of chromosome anomalies on the fertility of the different breeds of domestic dogs should be explored.

\section{REFERENCES}

de Grouchy J, Roubin M, Passage E (1964) Microtechnique pour l'étude des chromosomes humains à partir d'une culture de leucocytes sanguins. Ann Génét 7, 45-46

Di Berardino D, Lioi MB, Iannuzzi L (1985) Identification of nucleolus organizer chromosomes in cattle (Bos taurus L) by sequential silver staining + RBA banding. Caryologia $38,95-102$

Howard-Peebles PN, Pryor JC (1980) The R-banding pattern of the canine karyotype. $J$ Hered 71, 361-362

Mayr B, Schweizer D, Schleger W (1983) Characterization of the canine karyotype by counterstain-enhanced chromosome banding. Can J Genet Cytol 25, 616-621 